connective tissue of the submucosa beneath the lesion,3) perform EMR to dissect the lesion when its diameter smaller than the endoloops. Clinical data of both groups, such as LST size, LST location, endoscopy procedures, procedure time, en bloc resection rate, and post-SESD complications, were retrospectively analyzed.

Results 102 patients were involved in the study, SESD group involved 54 patients and ESD group involved 48 patients. The procedure time of SESD group was shorter than that of ESD group $(\mathrm{P}=0.32)$. There was no significant difference in the en bloc resection rate (SESD 100\% vs.ESD 98\%, $\mathrm{P}>0.05)$. Complication rates such as intraoperative perforation rate and postoperative bleeding rate also showed no statistic difference between two groups, so did recurrence rate. $(\mathrm{P}>0.05)$.

Conclusions Simplified Endoscopic Submucosal Dissection was an effective and safe therapy for colorectal LSTs.

\section{IDDF2020-ABS-0097 STUDY ON THE INFLUENCING FACTORS OF ERCP TREATMENT OF BENIGN BILIARY STRICTURE RECURRENCE}

Li-fang Ye*, Wen-hui Tan. Guangdong Second Provincial General Hospital, China

10.1136/gutjnl-2020-IDDF.77

Background To investigate the influencing factors of benign biliary stricture recurrence after ERCP treatment.

Methods A total of 100 patients of biliary stenosis with endoscopic biliary stent implantation from February 2017 to March 2020 were enrolled in our study. Patients were divided into the recurrence group and recurrence group according to incision re-stenosing within one year after removing the stents. The influencing factors of postoperative recurrence were compared between the two groups.

Results Hilar biliary stricture, common bile duct incision $+\mathrm{T}$ tube drainage, length of bile duct stenosis, and proximal dilation of biliary stricture were important high-risk factors for benign biliary stricture recurrence after endoscopic biliary stent placement $(\mathrm{P}<0.05)$.

Conclusions There are various factors that affect the recurrence of benign biliary stricture after ERCP treatment. It helped reduce the recurrence rate through risk factors intervention.

\section{IDDF2020-ABS-0098 DELAYED NURSING OF TRANSANAL ENDOSCOPIC MICROSURGERY FOR RECTAL MALIGNANT TUMOR}

Fang Wang*. Guangdong Second Provincial General Hospital, China

\subsection{6/gutjnl-2020-IDDF.78}

Background To explore the effect of delayed nursing of transanal endoscopic microsurgery for rectal malignant tumor.

Methods Patients underwent transanal endoscopic microsurgery for rectal malignant tumor in our hospital from January 2017 to February 2019 were enrolled in this study, and randomly divided into observation group and control group. The control group was performed with usual care, while the observation group was performed with delayed nursing. Peri-operative bleeding, postoperative anus exhausting time, hospital stays, the severity of fecal incontinence (Wexner Sores) and $\mathrm{Xu}$ Zhong Sores were analyzed and compared.

Results Postoperative anus exhausting time and hospital stays of observation group were shorter in the observation group than the control group, and peri-operative bleeding was also less in the observation group. The severity of fecal incontinence (Wexner Sores) and Xu Zhong Sores were both higher in the observation group.

Conclusions Delayed nursing was effective in transanal endoscopic microsurgery for rectal malignant tumor, for shortening postoperative anus exhausting time and hospital stays, and reduce peri-operative bleeding.

\section{IDDF2020-ABS-0099 NURSING MANAGEMENT STRATEGY IN ENDOSCOPY CENTER DURING THE EPIDEMIC PERIOD OF COVID-19}

Fang Lv*. Guangdong Second Provincial General Hospital, China

\subsection{6/gutjnl-2020-IDDF.79}

Background To explore the nursing management strategy in the endoscopy center during the epidemic period of COVID19.

Methods 2415 patients underwent endoscopy in our center from February 10th 2019 to March 27th 2019 were taken as control group, while 308 patients underwent endoscopy during February 10th 2020 to March 27th 2020 were taken as observation group. In our study, we reduced medical occupational exposure and avoid cross-infection during the epidemic period through reducing the number of patients, performing strict sterilization, isolation and preventive measures, implementing personnel training and assessment of both medical workers and patients, establishing emergency plans.

Results None of medical workers infected with COVID-19. Patients in the observation group were significantly decreased compared to the control group. The sterilization frequency of endoscopic machines, probes of blood oxygen monitor, examining beds and gastrointestinal endoscopes was increased markedly in the observation group. Preventive measures and the mastery of epidemiology were also significantly improved in the observation group.

Conclusions It is effective to prevent and control COVID-19 in endoscopy center during the epidemic period through reducing the number of patients, performing strict sterilization, isolation and preventive measures, implementing personnel training and assessment of both medical workers and patients, establishing emergency plans.

\section{IDDF2020-ABS-0101 IMPACT OF OPTIMAL TIMING OF EARLY PRECUT SPHINCTEROTOMY ON THE RISK OF ENDOSCOPIC RETROGRADE CHOLANGIOPANCREATOGRAPHY RELATED ADVERSE EVENTS: A SYSTEMATIC REVIEW AND META-ANALYSIS}

Carlos Paolo Dimatatac Francisco*, Nikko Theodore Raymundo, Enrik John Aguila, Jonard Co. St. Luke's Medical Center - Bonifacio Global City, Institute of Digestive and Liver Diseases, Philippines

10.1136/gutjnl-2020-IDDF.80 